\title{
Temporally Regularized Non-local De-noising and Its Application to Cardiac Longitudinal MR Images
}

\author{
Chaolu Feng, ${ }^{1, a, *}$ Junchi Lu ${ }^{1, b}$ and Dazhe Zhao ${ }^{1, c}$ \\ ${ }^{1}$ School of Computer Science and Engineering, Northeastern University, Shenyang, Liaoning, China \\ afengchaolu@cse.neu.edu.cn, blujunchi9@163.com, ${ }^{c}$ zhaodazhe@mail.neu.edu.cn \\ *corresponding author
}

Keywords: Non-Local De-Noising, Longitudinal Images, Temporal Regularization, CUDA Acceleration

\begin{abstract}
Longitudinal image analysis has become a hot research topic due to the progress of medical image processing and analysis. However, noises generally exist in most of medical images, which will negatively affect statistical characteristics of the image intensities and therefore weaken the contrast between different organs to further result in difficulties to image processing methods. In this paper, traditional local de-noising methods are first reviewed with their disadvantages in introducing new artifacts being revealed. On the contrary, non-local de-noising eliminates image noises while intact image structure information is preserved. Therefore, an improved non-local method is then proposed by incorporating temporal information of longitudinal images into the formula of non-local de-noising. Finally, the proposed method is applied to eliminate noises from cardiac longitudinal MR images. The proposed method searches similar pairs in the whole image space rather than in local areas, which is particularly different from traditional methods where only neighborhood intensities are used. The similarities are considered as weightings which are used to estimate the intensity by weighted average of all similar pairs. Experimental results show that the proposed method can effectively eliminate noises from cardiac longitudinal images without weakening boundaries of the images. To improve time performance, the proposed method is accelerated using CUDA and $150 \times$ improvement is obtained.
\end{abstract}

\section{Introduction}

With the rapid development of imaging technology and the progress of computers in computing and storing, medical imaging modalities such as MR, CT and ultrasound have been significantly improved in speed. Therefore, it is possible to scan the same pathological tissue regularly and store the images orderly in order to further analyze the development process of the disease. In the literature, the images of the same human tissue acquired at different time points are named as Longitudinal Image Series [1]. In the wake of progresses in medical image processing and analysis, longitudinal analysis has gradually attracts interests of researchers and scientists in this field [2].

Due to the influence of acquisition environment, there are generally noises in images acquired by medical devices, which necessarily affects statistical property of image intensities, weakens tissue contrasts, and presents challenges to image processing algorithms. Therefore, it is the problem that image processing and analysis algorithms have to face with that how to effectively eliminate the negative effects of noises [3]. Conventional de-noising methods locally treat tiny details and noises equally, which may distort structure information and therefore results in additional artifacts besides noise elimination [4]. Non-Local de-noising methods can effectively eliminate image noise while preserving structure information completely. However, existing non-local de-noising methods do not consider the multitemporal property of longitudinal image series. 
In this paper, a temporally regularized non-local de-noising method is proposed. Different from looking for similar pairs in images acquired at discrete time, the proposed method seeks similar pairs at all time points. The similarity degree is defined as contrast weight, and the weighted mean of all pairs is used to estimate the corrected intensity.

The rest of this paper is organized as follows. In Section II, we briefly review relevant work, give implementation principles of traditional methods, and analyze their pros and cons. In Section III, we first introduce traditional non-local means de-noising and then give details of the proposed temporally regularized non-local de-noising method. We apply the proposed method to eliminate noise in cardiac longitudinal MR images and analyze the differences from traditional non-local de-noising method in Section IV. Discussion and conclusions are given in Section V.

\section{Related work}

Image noise is usually signal dependent and independently distributed at different locations of the image domain. Generally, the existing de-noising methods are based on the assumption that the noise is oscillatory and the image is smooth or piecewise smooth. Therefore, the purpose of de-noising is to separate smooth or piecewise smooth images from oscillating signals.

\section{A. filtering in frequency domain}

Given an image $I(\mathbf{x}, t)$ acquired at time point $t$ defined on the domain $\Omega \subset R^{m}$ where $m$ is dimensionality of the image, intensities of the image are viewed as a map from $\Omega$ to $R$, i.e., $I: \Omega \rightarrow R$. To eliminate noise, frequency domain methods first map time-domain image $I(\mathbf{x}, t)$ to frequency space using Fourier transform, given by

$$
F(\mathbf{u}, t)=\int_{\mathbf{x} \in \Omega} I(\mathbf{x}, t) e^{-j 2 \pi \mathbf{x}} d \mathbf{x} .
$$

Then, given a filtering function $H(\mathbf{u}, t)$, which is low pass, intermediate pass or high pass, de-noising in the frequency domain is written as $G(\mathbf{u}, t)=F(\mathbf{u}, t) H(\mathbf{u}, t)$. Finally, the noise free signal $G(\mathbf{u}, t)$ is transformed back to the time domain by the inverse Fourier transform, given by

$$
\hat{I}(\mathbf{x}, t)=\int_{-\infty}^{+\infty} G(\mathbf{u}, t) e^{j 2 \pi \mathbf{u} x} d \mathbf{u} \text {. }
$$

Disadvantages of this kind of method are that the de-noising result depends on predetermined pass functions, and it costs extra time to transform the image from time-domain to frequency domain or vice versa [5].

\section{B. Local smooth filtering}

Traditional time-domain de-noising methods usually estimate new intensities using the ones in local regions. Mean and median filtering are two typical representatives of local smooth de-noising [6]. The former is based on neighborhood averaging and is therefore suitable for removing particle noise existing in images. Gaussian filtering is one typical representative of mean filtering where intensities in the neighborhood are given predetermined weights. The weights are location dependent, defined by

$$
G_{\sigma}(\mathbf{x})=\frac{1}{2 \pi \sigma^{2}} e^{-\frac{|\mathbf{x}|^{2}}{2 \sigma^{2}}}
$$

where $\sigma$ is a parameter used to control the standard deviation. De-noising is then performed by convoluting the image $I(\mathbf{x}, t)$ with $G_{\sigma}(\mathbf{x})$, defined by $\widetilde{I}=I * G_{\sigma}$ where $*$ is the convolution operator. Although neighborhood averaging methods can suppress noise, they will also blur the image due to the averaging operation and the degree of fuzziness is directly relevant to the neighborhood radius.

Median filtering is another kind of regional smoothing filtering which does not consider statistical characteristics of image intensities. Its basic principle is to replace the intensity at a location of the image with the median of its surroundings. Median filtering is effective for salt and pepper noise. But tiny tissues are also removed as noise and the image will therefore be blurred. 


\section{Non-local denoising}

In fact, human tissues may also manifest intensity oscillation and non-smoothness. Moreover, there may be low frequency components in white noise. Therefore, it will release new challenges to image processing and analysis methods if image noise was removed depending only on smoothness operations. In addition, the traditional de-noising methods depend on local smooth filtering, which will distort structure information and bring new artifacts.

\section{A. Non-local means}

Besides neighborhoods, intensities at other locations of the image are also useful for noise removal in practice. Therefore, Buades et al. proposed non-local mean de-noising (NLMD) in [7] where intensity similarity is matched in the entire image domain rather than in a local region. Further, the similarities are considered as weights used to estimate corrected intensities with weighting average [8]. Noise removal on the image $I(\mathbf{x}, t)$ acquired at time point $t$ can be defined by

$$
\bar{I}(\mathbf{x}, t)=\frac{1}{C_{t}(\mathbf{x})} \int_{\Omega} W_{t}(\mathbf{x}, \mathbf{y}) I(\mathbf{y}, t) d \mathbf{y}
$$

where

$$
W_{t}(\mathbf{x}, \mathbf{y})=e^{-\frac{\left(G_{\sigma}^{*}|I(\mathbf{x}+, t)-I(\mathbf{y}+, t)|^{2}\right)(0)}{h^{2}}}
$$

and

$$
\begin{aligned}
& \left(G_{\sigma} *|I(\mathbf{x}+., t)-I(\mathbf{y}+., t)|^{2}\right)(0) \\
& =\int G_{\sigma}(\mathbf{z})|I(\mathbf{x}+., t)-I(\mathbf{y}+., t)|^{2} d \mathbf{z}
\end{aligned} .
$$

Note that $h$ is a control parameter and $C_{t}(\boldsymbol{x})$ is a normalization factor defined by

$$
C_{t}(\mathbf{x})=\int_{\Omega} e^{-\frac{\left(G_{\sigma}^{* * I}(\mathbf{x}+, t)-\left.I(\mathbf{z}+, t)\right|^{2}\right)(0)}{h^{2}}} d \mathbf{z} \text {. }
$$

As described in Eq.(4), image intensity $I(\mathbf{x}, t)$ is replaced by the weight mean of $I(\mathbf{y}, t)$ and the weightings are great if intensity distribution in the Gaussian window around $\mathbf{y}$ is similar to that in the window around $\mathbf{x}$ and vice versa. Obviously, self-similarity of the image is taken into account to eliminate the image noise and the local regional features of the image will therefore not be destroyed.

B. Temporally Regularized Non-local means

In order to eliminate noises in longitudinal image series acquired at different time and ensure the local characteristics of the images maintained, the above mentioned NLMD can be used to remove noise from images at each time point separately. The disadvantage is that it does not take into account the correspondence of the same location at different time. Therefore, temporally regularized non-local means is proposed in this section. Similar to Eq.(4), suppose the series consist of images at T time points, image intensity $I(\mathbf{x})$ is replaced by the weight mean of $I(\mathbf{y})$, defined by

$$
\breve{I}(\mathbf{x})=\frac{1}{C(\mathbf{x})} \int_{\Omega} W(\mathbf{x}, \mathbf{y}) I(\mathbf{y}) d \mathbf{y}
$$

where

$$
\begin{aligned}
& W(\mathbf{x}, \mathbf{y})=e^{-\frac{\left(G_{\sigma} * I(\mathbf{x}+.)-\left.I(\mathbf{y}+.)\right|^{2}\right)(0)}{h^{2}}} \\
& \text { and } \\
& \left(G_{\sigma} *|I(\mathbf{x}+.)-I(\mathbf{y}+.)|^{2}\right)(0) \\
& =\int G_{\sigma}(\mathbf{z})\left(\sum_{t=1}^{T} \mid I(\mathbf{x}+., t)-I\left(\mathbf{y}+.,\left.t\right|^{2}\right) d \mathbf{z}\right.
\end{aligned}
$$

Note that $C(\mathbf{x})$ is a normalization factor defined by

$$
C(\mathbf{x})=\int_{\Omega} e^{-\frac{\left.\left(G_{\sigma}^{*} \mid I(\mathbf{x}+)\right)-\left.I(\mathbf{z}+.)\right|^{2}\right)(0)}{h^{2}}} d \mathbf{z}
$$


As mentioned earlier, NLMD considers that the intensity at each location can be linked to all the others. That is, if resolution scale of the image is $\mathrm{P}$, time complexity of NLMD is $\mathrm{O}^{3}\left(\mathrm{P}^{3}\right)$ and the proposed temporally regularized NLMD is $\mathrm{O}\left(\mathrm{T} \times \mathrm{P}^{3}\right)$. In order to improve time performance, the number of locations taken into account in the weighted average is practically restricted in a neighborhood of size $M$ and the local similarity is further restricted in the size of $d$ (known as search window in the literature).

\section{Experimental result}

To compare the proposed method visually with traditional frequency domain based, local, and non-local methods, and verify the advantages and disadvantages, a series of cardiac MR images collected at 20 time points with a resolution of $256 \times 256 \times 11$ are chosen. For the convenience of comparison, we show results of the comparable methods on one of the same slice of the images series at end diastole. As shown in Fig. 1, low pass filtering are able to reduce the image noise. But they will weaken boundaries and blur the image. High pass filtering sharpens the image after de-noising, making the boundary more obvious, but some details of the image are lost. Median filtering is just in the middle of the above two characteristics.
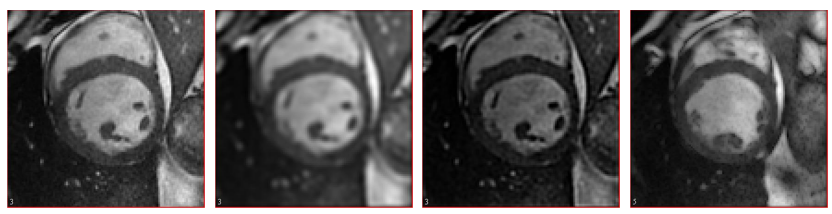

(a) original

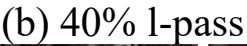

(c) $10-70 \%$ m-pass (d) 30\% h-pass
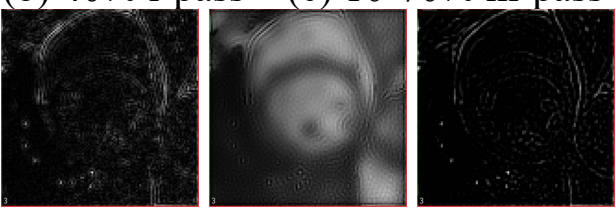

(e) noise from (b) (f) noise from (c) (g) noise from (d)

Fig.1 Results of frequency filters on one slice of cardiac MR image.

Results of smooth filtering on the same image slice are given in Fig. 2. All the filtering methods estimate noise using neighbors of size 5. As shown in Fig. 2, it can be seen that the filtering methods will weaken boundaries of the cardiac left ventricle in varying degrees. Median filtering has little influence on boundaries of the cardiac left ventricle, but it changes the original grayscale distribution of the image.

In order to verify that the NLMD method does not destroy local features of image where noises are eliminated effectively, and prove correctness of the proposed temporally regularized NLMD (which we call $3 \mathrm{D}+\mathrm{T}$ in this experiment), results of non-local filtering on one slice of the cardiac longitudinal MR images are given in Fig. 3. Note that the image is the same one used in Fig. 1 and Fig. 2. For the convenience of comparison, the parameters are set as follows $M=7, d=3, h=10$. As shown in Fig. 3, besides noises are removed from the image, the proposed temporally regularized NLMD method does not change the gray distribution of the original image and boundaries of the cardiac left ventricle are not weaken by the method. Moreover, results of the proposed method are better than those of the traditional NLMD in its 2-dimensional and 3-dimensional versions. 


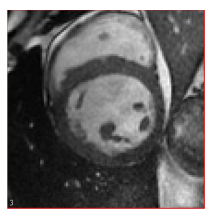

(a) original

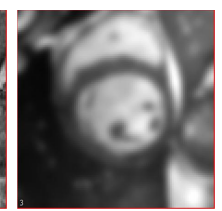

(b) Gaussian
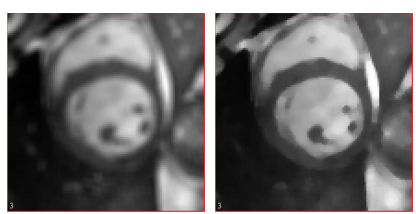

(c) mean (d) median
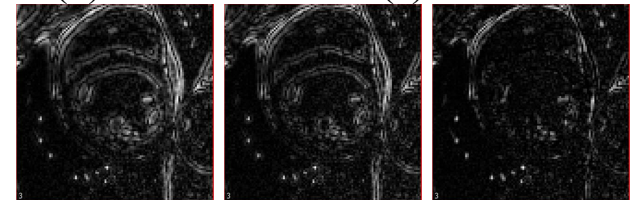

(e) noise from (b) (f) noise from (c) (g) noise from (d)

Fig. 2 Results local filtering on one slice of cardiac MR image.
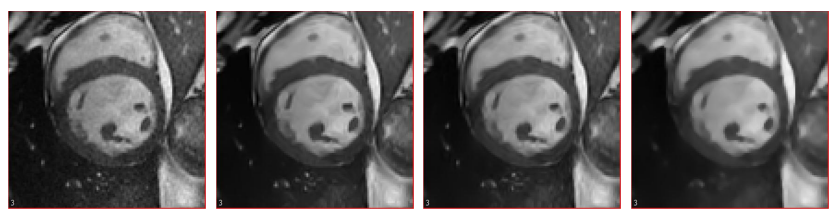

(a) original

(b) 2D NLMD

(c)3D NLMD

(d)3D+T NLMD


(e) noise from (b) (f) noise from (c) (g) noise from (d)

Fig. 3 Results non-local filtering on one slice of cardiac MR image.

\section{Discussion and conclusions}

A temporally regularized non-local de-noising has been proposed. We applied it to remove noises for cardiac longitudinal MR images. Experimental results show its correctness and advantages over state-ofthe-art methods. As reported in [9], it costs 6 hours for traditional NLMD to remove noises in its 3dimensional version from images in resolution of $181 \times 217 \times 181$ with parameters $M=5$ and $d=1$. To improve the time performance of the proposed method, we accelerate it using CUDA in the implementation with a similar strategy exploited in [10] and obtain 150 times speedup.

\section{Acknowledgment}

The first author would like to thank his wife Yang Hu for her helpful suggestions on the writing of this paper.This work was supported by the National Natural Science Foundation of China under grant 61602101, the Fundamental Research Funds for the Central Universities of China under grant N161604003, and ShenYang Science and Technology Program under grant 17-134-8-00.

\section{References}

[1] Feng C, Zhao D, Huang M. Segmentation of longitudinal brain MR images using bias correction embedded fuzzy c-means with non-locally spatio-temporal regularization[J]. Journal of Visual Communication and Image Representation, 2016, 38: 517-529

[2] Gerig G, Fishbaugh J, Sadeghi N. Longitudinal modeling of appearance and shape and its potential for clinical use [J]. Medical Image Analysis, 2016, 33: 114-121.

[3] Shao L, Yan R, Li X, et al. From heuristic optimization to dictionary learning: a review and comprehensive comparison of image denoising algorithms [J]. IEEE Transactions on Cybernetics, 2014, 
44(7): 1001-1013.

[4] Li Q. Research on image denoising and smothing methods based on nonlocal sparsity [D], Jinan: Shandong University, 2016.

[5] Feng C. Research on key algorithms for segmentaion and visualization of cardiac tissues [D], Shenyang: Northeastern University, 2014.

[6] Chen C, Ni J, Huang J. Blind detection of median filtering in digital images: a difference domain based approach [J], IEEE Transactions on Image Processing, 2013, 22(12):4699-710.

[7] Buades A, Coll B, Morel J M. A non-local algorithm for image denoising [C], IEEE Computer Society Conference on Computer Vision and Pattern Recognition, 2005, 2: 60-65.

[8] Tracey B H, Miller E L. Nonlocal means denoising of ECG signals [J], IEEE Transactions on Biomedical Engineering, 2012, 59(9):2383-6

[9] Coupé P, Yger P, Barillot C. Fast non local means denoising for 3D MR images [C], Medical Image Computing and Computer-Assisted Intervention, 2006, 9(Pt 2):33-40.

[10] Feng C, Zhao D, Huang M. Image segmentation using CUDA accelerated non-local means denoising and bias correction embedded fuzzy c-means (BCEFCM) [J]. Signal Processing, 2016, 122: $164-189$. 